\title{
Kültürel Mirasın Kullanıcı Merkezli Etkileşimli Deneyimi için Bütünsel Bir Uygulama: 3B Baskı, Artırılmış Gerçeklik ve Web Tabanlı Görselleştirme Kombinasyonu
}

\author{
Ahmet Uslu ${ }^{1 *}$ \\ 1* Kütahya Dumlupınar Üniversitesi, Tavşanlı Meslek Yüksekokulu, Mimarlık ve Şehir Planlama Bölümü, Kütahya, Türkiye, (ORCID: 0000-0001-8745-423X), \\ ahmet.uslu1@dpu.edu.tr
}

(1st International Conference on Applied Engineering and Natural Sciences ICAENS 2021, November 1-3, 2021)

(DOI: 10.31590/ejosat.1014904)

ATIF/REFERENCE: Uslu, A. (2021). Kültürel Mirasın Kullanıcı Merkezli Etkileşimli Deneyimi için Bütünsel Bir Uygulama: 3B Baskı, Artırılmış Gerçeklik ve Web Tabanlı Görselleştirme Kombinasyonu. Avrupa Bilim ve Teknoloji Dergisi, (28), 1291-1295.

\section{$\ddot{O} \mathbf{z}$}

Günümüzde donanım, yazılım, 3B dijital model üretimi ve sunumu alanındaki son teknolojik gelişmeler, insanların kültürel mirasa erişimini sağlamak için çeşitli firsatlar sunmaktadır. Fotogrametri, 3B dijital modelleme, 3B baskı, artırılmış gerçeklik ve web tabanlı sunum teknolojileri, kültür varlıkları ile ilgili olarak kullanıcı etkileşimini geliştirme bakımından önemli avantajlar sağlamaktadır. Bu çalışmada, fotogrametrik modellere dayalı 3B baskı, artırılmış gerçeklik ve web tabanlı görselleştirme uygulamaları için yenilikçi bir yaklaşım ve iş akışı geliştirerek kültürel miras bağlamında kullanıcı merkezli sanal, sürükleyici ve etkileşimli bir deneyim yaşatılması amaçlanmıştır. Çalışmanın sonuçları, 3B baskı, artırılmış gerçeklik ve web tabanlı görselleştirme sinerjisinin kültürel mirasın etkileşimli olarak deneyimlenmesi ve ayrıntılı keşfi açısından büyük bir imkân sağladığını göstermiştir. Çalışma kültürel mirasın korunması, gelecek nesillere aktarılması ve sanal erişilebilirliği için insan-yenilikçi teknoloji etkileşimine değerli katkılar sunmaktadır.

Anahtar Kelimeler: Kültürel miras, 3B modelleme, 3B baskı, Artırılmış gerçeklik, Web tabanlı görselleştirme.

\section{A Holistic Application for a User-Centered Interactive Experience of Cultural Heritage: A Combination of 3D Printing, Augmented Reality and Web-Based Visualization}

\begin{abstract}
Today, the latest technological developments in the field of hardware, software, production and presentation of 3D digital models provide various opportunities to ensure people's access to cultural heritage. Photogrammetry, 3D digital modeling, 3D printing, augmented reality and web-based presentation technologies provide significant advantages in terms of improving user interaction in relation to cultural assets. In this study, based on photogrammetric model, 3D printing, augmented reality and web-based visualization of cultural heritage in the context of an innovative approach for applications and workflow by improving user-centric virtual, immersive and interactive experience that is intended to maintain. The results of the study have shown that the synergy of 3D printing, augmented reality and web-based visualization provides a great opportunity for interactive experience and detailed exploration of cultural heritage. The study makes valuable contributions to human-innovative technology interaction for the protection of cultural heritage, its transfer to future generations and its virtual accessibility.
\end{abstract}

Keywords: Cultural heritage, 3D modelling, 3D printing, Augmented reality, Web-based visualization.

*Sorumlu Yazar: ahmet.uslu1@dpu.edu.tr 


\section{Giriş}

Günümüzde donanım, yazılım, 3B dijital model üretimi ve sunumu alanındaki son teknolojik gelişmeler, kültürel mirasın uzaktan keşfedilmesine imkân sağlamaktadır (FernándezPalacios ve ark., 2017). Lazer tarama ve fotogrametri gibi gerçekliğe dayalı 3B ölçme teknikleri kullanılarak belgeleme, arkeolojik ve mimari analizler, görselleştirme, eğitim amaçlı müze sergileri ve sanal turizm için ayrıntılı ve foto-gerçekçi $3 \mathrm{~B}$ dijital modeller üretebilmektedir (Fernández-Palacios ve ark., 2017). 3B dijital modellerin artırılmış gerçeklik, sanal gerçeklik, karma gerçeklik, 3B baskı ve web tabanlı görselleştirme gibi etkileşimli ortamlara entegrasyonu ile birlikte nesnelere alternatif erişim biçimi ve kullanıcılara sürükleyici yeni deneyimler sağlanabilir (Putra ve ark., 2016).

Son yıllarda kültürel miras bağlamında fotogrametri, artırılmış gerçeklik, sanal gerçeklik, karma gerçeklik, 3B baskı ve web tabanlı görselleştirme sinerjisi üzerine yapılan araştırmalar hız kazanmıştır. Uslu ve Uysal (2017), araştırmalarında sanal müze uygulaması için arkeolojik eseri fotogrametri tekniği ile 3B modellemişler ve 3B modeli HTML 5-WEBGL kullanarak internet tarayıcısı üzerinde görselleştirmiş̧lerdir. Aziz ve Siang (2014), UNESCO Dünya Mirası Listesinde yer alan Melaka'da kültürel mirasın korunması için bütünsel bir uygulama olarak artırılmış gerçeklik ve sanal gerçeklik kombinasyonunu gerçekleştirmişlerdir. Uslu ve Uysal (2020), bir arkeolojik eserin etkileşimli keşfi için fotogrametri tekniği ile mobil artırılmış gerçeklik ve web tabanlı sunum teknolojileri arasında bir kombinasyon oluşturmuşlardır. Uslu ve Uysal (2021), araştırmalarında sosyal medya platformu Flickr'da paylaşılan fotoğrafları kullanarak Afrodisias Antik Kenti'nde bulunan Tetrapylon yapısını fotogrametri tekniği ile 3B modellemişler ve 3B modeli dijital erişim için sanal küre üzerinde web tabanlı görselleştirmişlerdir. Bozzelli ve ark. (2019), araştırmalarında kültürel mirasın kullanıcı merkezli etkileşimli deneyimi için entegre bir artırılmış gerçeklik ve sanal gerçeklik projesi olan ArkaeVision'1 kullanıma sunmuşlardır. ArkaeVision projesi ile daha ilgi çekici ve kültürel açıdan nitelikli bir kullanıcı deneyimi sunarak kültürel mirasın keyfini çıkarmanın yeni bir yolunu sağlamayı amaçlamışlardır. Jung ve tom Dieck (2017), çalışmalarında kültürel miras alanlarındaki zengineliştirici ve sürükleyici ziyaretçi deneyimi için artırılmış gerçeklik, sanal gerçeklik ve 3B baskı kombinasyonunu uygulamışlardır.

Literatür araştırması neticesinde değer yaratma perspektifinden kültürel mirasın kullanıcı merkezli etkileşimli deneyimi için bütünsel bir uygulama olarak 3B baskı, artırılmış gerçeklik ve web tabanlı görselleştirme kombinasyonu üzerine ülkemizde yapılmış herhangi bir bilimsel araştırmaya rastlanılmamıştır. Bu çalışmada, fotogrametrik modellere dayalı 3B baskı, artırılmış gerçeklik ve web tabanlı görselleştirme uygulamaları için bir yaklaşım ve iş akışı geliştirerek kültürel miras bağlamında kullanıcı merkezli sanal, etkileşimli ve sürükleyici bir deneyim oluşturulması amaçlanmıştır. İş akışı, Kütahya ili, Merkez ilçesi, Zafer Meydanında yer alan tarihi Hürriyet Çeşmesi'ne uygulanmıştır

\section{Materyal ve Metot}

\section{1. Çalışma Alanı ve Çalışmanın Materyalleri}

Çalışmada Kütahya ili, Merkez ilçesi, Zafer Meydanında yer alan tarihi Hürriyet Çeşmesi model olarak seçilmiştir (Şekil 1).

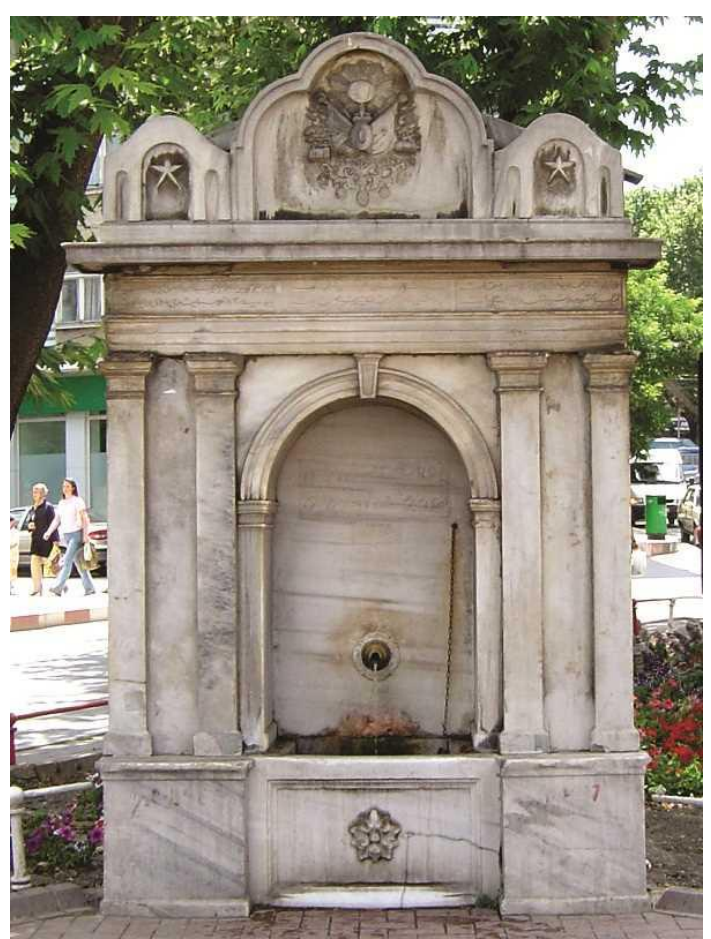

Şekil 1. Hürriyet Çeşmesi

Hürriyet Çeşmesi, üzerinde bulunan kitabesine göre II. Abdülhamid döneminde 1909 yılında inşa edilmiştir. Batılı ve Eklektik üslupta inşa edilen dikdörtgen plandaki Hürriyet Çeşmesi, 3,40 m yüksekliğe, 2,05 m genişliğe ve $1 \mathrm{~m}$ derinliğe sahiptir (Verim 2019).

Çalışmada, Hürriyet Çeşmesi'nin fotoğraflarının çekimi için Samsung ST150 dijital fotoğraf makinesi, çeşme üzerinde belirlenen kontrol noktalarının koordinat değerlerini ölçmek için Spectra Focus 6 totalstation cihazı, fotogrametrik değerlendirme ve $3 \mathrm{~B}$ modelleme için Agisoft PhotoScan yazılımı, web tabanlı görselleştirme işlemleri için sanal küre platformu Cesium, artırılmış gerçeklik uygulaması için Augment (bulut tabanlı yazılım), 3B baskı öncesi gerekli kontrollerin ve düzenlemelerin yapılması için Autodesk Meshmixer yazılımı, 3B baskı parametrelerinin tanımlanması için Ultimaker Cura yazılımı ve son olarak 3B baskı işlemi için Prusa i3 3B yazıcı kullanılmıştır

\subsection{Yöntem}

Çalışmanın yöntemi verilerin toplanması, fotogrametrik değerlendirme, 3B modelleme, 3B baskı, artırılmış gerçeklik ve web tabanlı görselleştirme aşamalarından oluşmaktadır. Şekil 2' de çalışmanın yöntemi gösterilmiştir.

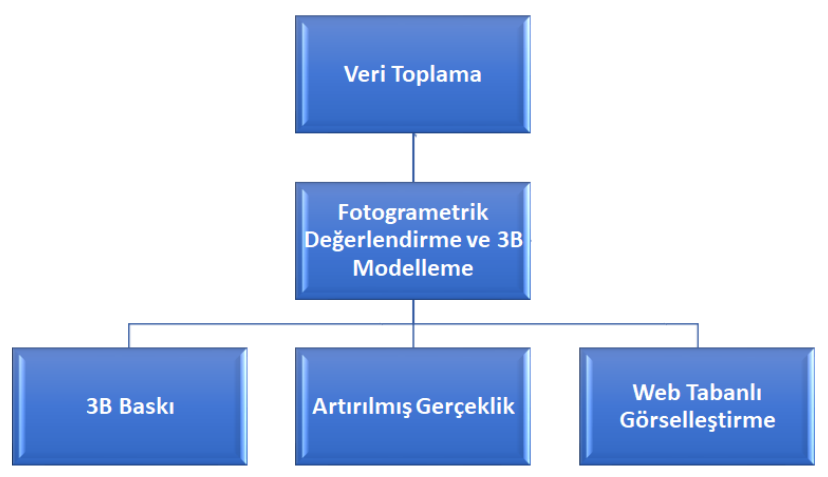

Şekil 2. Çalışmanın yöntemi. 


\section{Bulgular}

\subsection{Veri Toplama}

Fotogrametri tekniği kullanarak 3B modelleme işlemi için planlı jeodezik ölçüm ve fotoğraf çekimi yapılmalıdır (Uslu ve Uysal, 2017). Bu bağlamda tarihi çeşme üzerinde işaretlenen kontrol noktalarının koordinat değerlerini ölçmek için öncelikle çeşmeyi tüm yönleriyle kapsayan lokal koordinat sisteminde kapalı bir jeodezik ağ oluşturulmuştur. Daha sonra çeşme üzerinde homojen dağılımlı 18 adet karakteristik kontrol noktası belirlenmiştir. Kontrol noktalarının ölçümünde Focus 6 reflektörsüz totalstation cihazı kullanılmıştır. Totalstation cihazının lazeri kullanılarak açı ve mesafe ölçmeleri aracılığıyla koordinat değerleri elde edilmiştir. Kontrol noktalarının ölçümünde sonra yüksek doğruluk ve çözünürlükte $3 \mathrm{~B}$ model üretimi için Samsung ST150 dijital fotoğraf makinesi ile konvergent çekim esasları dikkate alınarak Hürriyet Çeşmesinin 105 adet fotoğrafı çekilmiştir. Fotoğrafların çekimi farklı açılardan, bindirmeli ve birbirlerine referans olacak şekilde gerçekleştirilmiş, kontrol noktalarının 105 adet fotoğrafta görünür ve seçilebilir olmasına dikkat edilmiştir.

\subsection{Fotogrametrik Değerlendirme ve 3B Model Oluşturma}

Fotogrametrik değerlendirme ve 3B modelleme işlemi için ilk olarak arazi çalışmaları aşamasında elde edilen veriler bilgisayar ortamına aktarılmıştır. Fotoğraf veri seti olarak 105 adet fotoğraf kullanılmıştır. 105 adet fotoğrafın tamamı, 77,389 adet bağlantı noktası kullanılarak otomatik olarak hizalanmıştır. Daha sonra 3B modelin ölçeklendirilmesi amacıyla arazi çalışmaları sırasında koordinat değerleri ölçülen 18 adet kontrol noktası nokta atmak suretiyle işaretlenmiştir. Son olarak Hürriyet Çeşmesinin sırasıyla 3B yoğun nokta bulutu $(3,629,106$ adet) (Şekil 3), 3B yüzeyler (226,812 yüzey) ve 3B doku kaplı modeli oluşturulmuştur (Şekil 4).

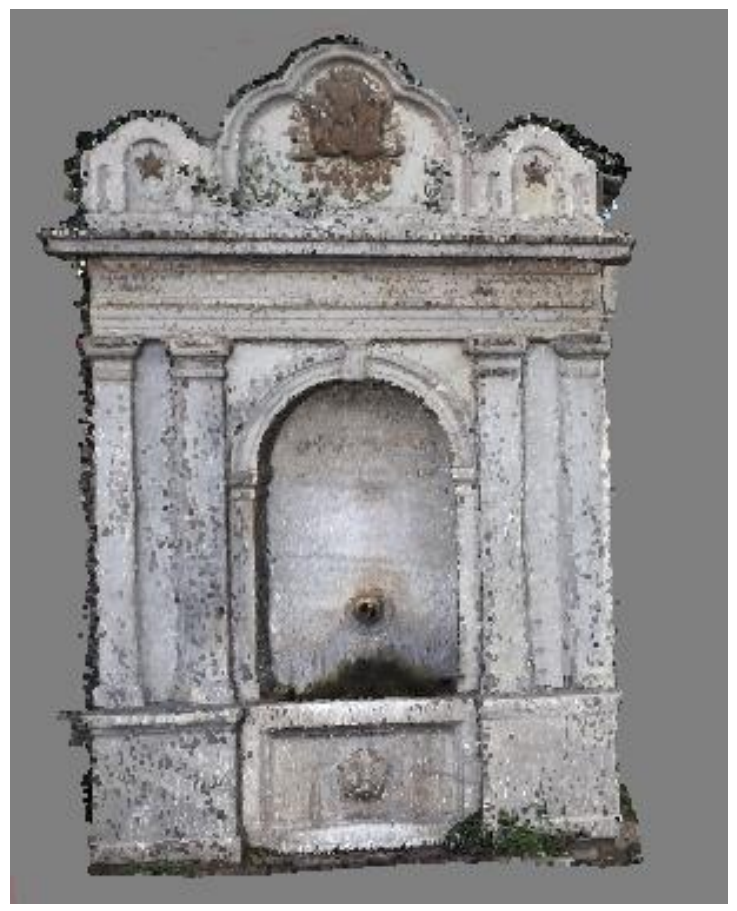

Şekil 3. Hürriyet Çeşmesi'nin 3B yoğun nokta bulutu.

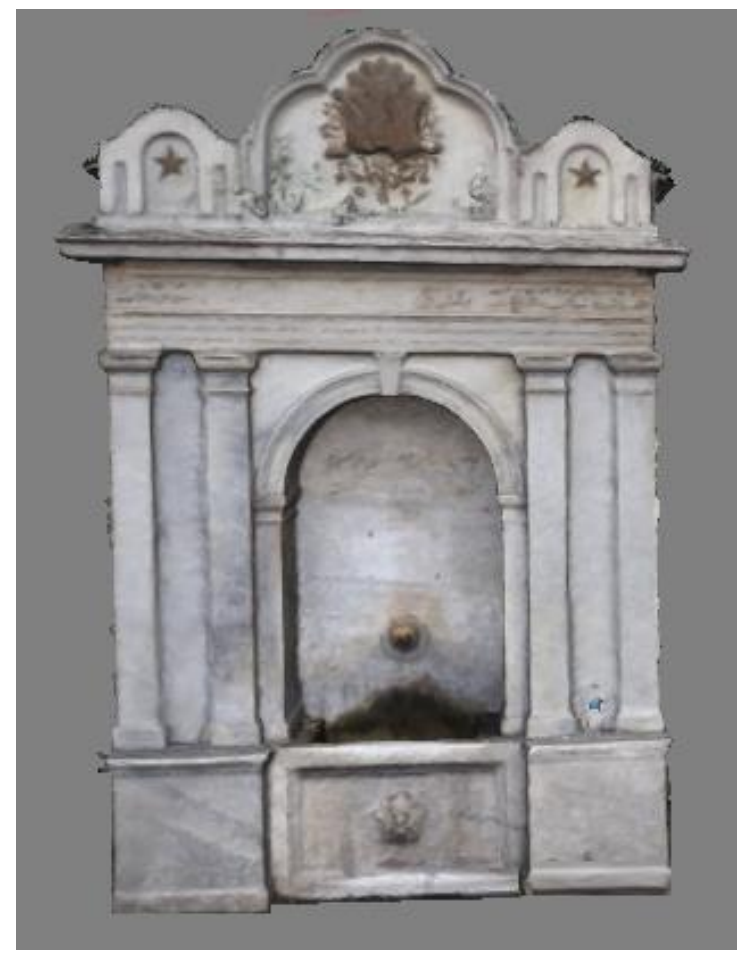

Şekil 4. Hürriyet Çeşmesi’nin doku kaplı 3B modeli.

Son olarak, 3B dijital model 3B baskı, artırılmış gerçeklik ve web tabanlı görselleştirme araştırmalarında kullanılmak amacıyla “.dae" uzantılı model dosyası olarak kaydedilmiştir.

\subsection{B Baskı Uygulaması}

3B bask1 öncesi gerekli kontrollerin ve düzenlemelerin yapılması amaciyla 3B model dosyası Autodesk Meshmixer yazılımına aktarılmıştır (Şekil 5).

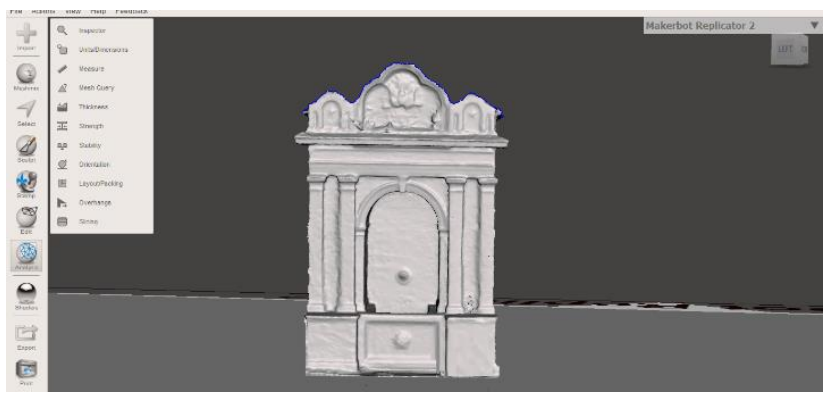

Şekil 5. Autodesk Meshmixer programında 3B baskı öncesi hazırlık işlemleri.

3B dijital modelin yüzeyi optimize edilerek model baskı işlemi öncesi hatasız hale getirilmiş ve ".stl" uzantılı dosya formatında dışarı aktarılmıştır. Bu işlemlerin ardından ".stl" uzantılı model dosyası 3B yazıcı programına (Ultimaker Cura) çağrılmış, yazdırma parametreleri tanımlanmış ve model yazdırma alanında uygun pozisyonda konumlandırılmıştır (Şekil 6). 


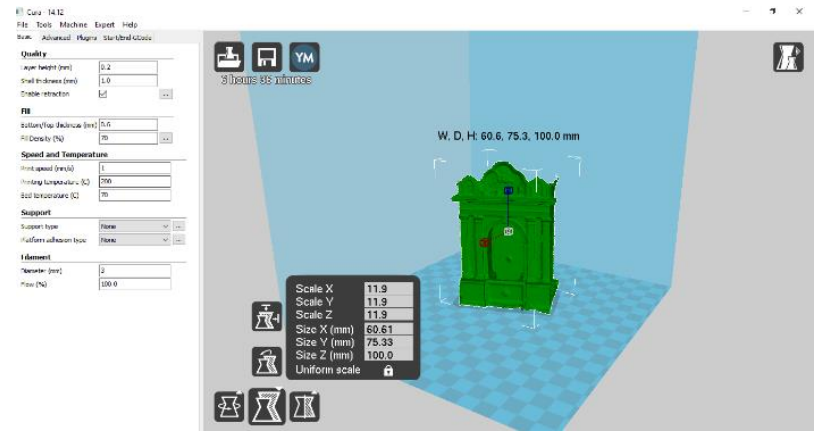

Şekil 6. Ultimaker Cura programında 3B baskı öncesi hazırlık işlemleri.

Yazdırma işlemi Prusa i3 3B yazıcıda $6.6 \mathrm{~cm}$ x $7.5 \mathrm{~cm} \times 10$ cm $(\mathrm{G}, \mathrm{D}, \mathrm{Y})$ ebatlarında, 50 mikron katman kalınlığı, \% 70 doluluk oranı ile 93 gram gri renkli PLA (Polilaktik Asit) malzeme kullanılarak 6 saat 36 dakikada gerçekleştirilmiştir. Yazdırma işlemi sonucunda imal edilen model Şekil 7'de görülmektedir.

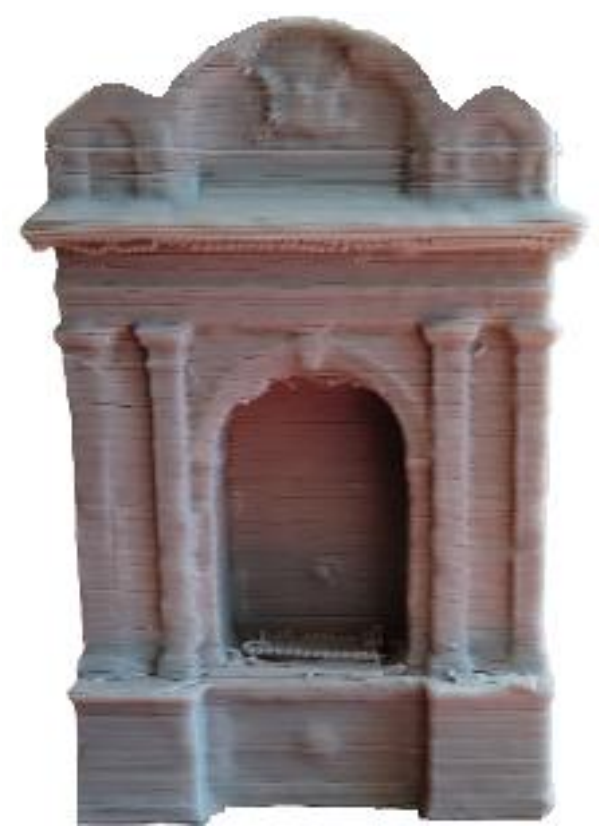

Şekil 7. Hürriyet Çeşmesinin 3B yazıcıdan üretilmiş çıktısı.

\subsection{Artırılmış Gerçeklik Uygulaması}

Artırılmış Gerçeklik, kültürel mirası yayma, koruma ve görselleştirme sürecini teşvik etmek için etkili bir araçtır. Sanal içeriğin fiziksel bir ortama etkileşimli ve gerçek zamanlı olarak eklendiği bir teknolojidir (Bostancu ve ark., 2015). Mobil artırılmış gerçeklik uygulaması bulut tabanlı bir yazılım olan Augment'te gerçekleştirilmiştir. Augment, kullanıcıların çeşitli amaçlar için oluşturdukları modellerini, mobil cihazlar aracılığıyla gerçek ortamda ve gerçek zamanlı olarak 3B görselleştirmelerine olanak sağlayan Artırılmış Gerçeklik platformudur (URL-1). 3B modelleme aşamasında oluşturulan Hürriyet Çeşmesi'nin “.dae” uzantılı model dosyası, kaplama ve materyal dosyaları ile birlikte Augment uygulamasına yüklenmiştir. İlgili dosyalar sisteme kaydedildikten sonra Augment uygulaması, Hürriyet Çeşmesi'nin tanıtıcı bilgilerine ve 3B modeline dijital erişim sağlayan bir QR kod tanımlamıştır. Mobil cihaz üzerinde kurulu bir Artırılmış Gerçeklik aplikasyonu aracılığıyla QR kod taratılarak Hürriyet Çeşmesi'nin 3B modeli mobil Artırılmış Gerçeklik uygulamasında görüntülenmiştir (Şekil 8).

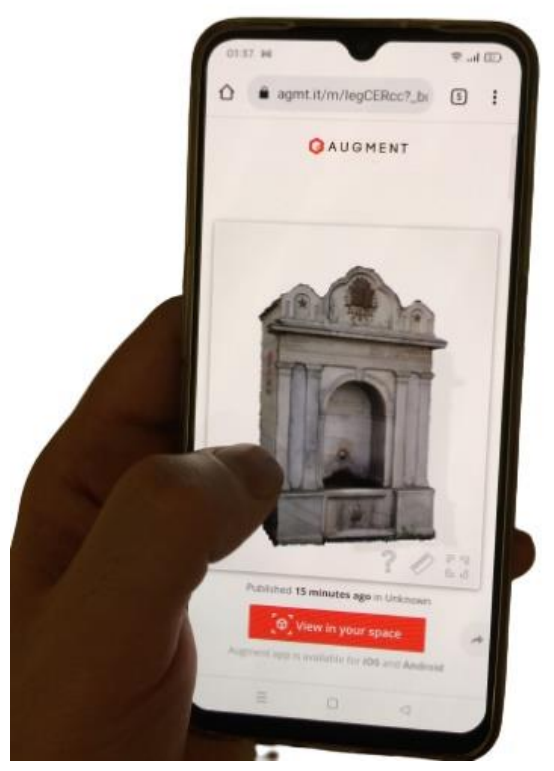

Şekil 8. Hürriyet Çesmesi'nin mobil Artırılmış Gerçeklik tabanlı görselleştirilmesi.

\subsection{Web Tabanlı Görselleştirme Uygulaması}

Web3B teknolojilerinde yaşanan son gelişmeler, kültür varlıklarının dijital olarak saklanmasında, korunmasında ve yaygınlaştırılmasında değerli katkılar sağlamıştır (Nishanbaev 2020). Sanal küre üzerinde $3 B$ görselleştirme, insanların sanal ortamda coğrafi verilerle doğrudan etkileşime girmesine imkân sağlayan, etkili bir görselleştirme ortamı olarak ifade edilmiştir (Nishanbaev 2020). Hürriyet Çeşmesi'nin sanal dünya üzerinde 3B gösterimi için açık kaynaklı Cesium Ion platformu kullanılmıştır. Cesium Ion, sanal dünya üzerinde çeşitli analizler için etkileşimli 3B haritalar oluşturmak amacıyla geliştirilmiş bir yazılımdır (URL-2). 3B dijital modelinin web tabanlı sunumu için model dosyası, kaplama ve materyal dosyaları kullanıcı hesabı ile Cesium Ion platformuna aktarılmıştır. Hürriyet Çeşmesi'nin 3B dijital modeli coğrafi referanslı olduğu için yerküre üzerindeki konumuna yerleştirilmiştir. Şekil 9'da Hürriyet Çeşmesi'ne ait 3B modelin Cesium Ion yazılımında web tabanlı sunumu gösterilmiştir.

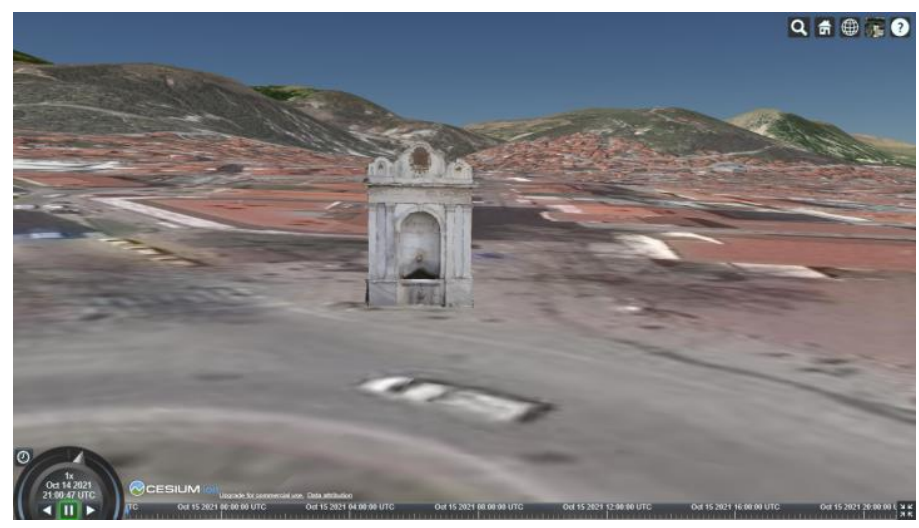

Şekil 9. Hürriyet Çesmesi'nin Cesium Ion yazılımında web tabanlı görselleştirilmesi.

Cesium Ion bilgisayarda yada mobil cihazlarda kullanıcıların sanal dünya üzerinde yakınlaştırma, kaydırma, döndürme ve gezinme gibi çoklu işlevleri gerçekleştirmelerini ve 3B görselleştirmeyi herhangi bir web sayfasına entegre etmelerini de mümkün kılmaktadır. 


\section{Tartışma ve Sonuç}

Bilgi teknolojilerindeki son gelişmeler, insanların kültürel mirasa erişimini sağlamak amacıyla çok çeşitli firsatlar sunmaktadır. Özellikle 3B bask1, web ortamında sunum ve artırılmış gerçeklik teknolojileri, kültür miras ile ilgili kullanıcı etkileşimini geliştirme bakımından önemli avantajlara sahiptir. Bu çalışmada, deneyimin bir parçası olarak birlikte değer yaratma ortamı oluşturmak için kültürel miras mekanlarında 3B bask1, artırılmış gerçeklik ve web tabanlı sunum teknolojilerini kullanma fikri kavramsallaştırılmıştır. Çalışmanın sonuçları, kültürel miras mekanları bağlamında çoklu teknolojilerin etkin kullanımının hem kültürel miras kuruluşları için hem de ziyaretçilerin ziyaret öncesi, yerinde ve ziyaret sonrası deneyimleri için birlikte değer yaratılmasına katkı sunduğunu göstermektedir. Çalışmada açıklanan yaklaşım sayesinde kültürel miras yerlerinin rekabet gücünü artırmasının yanı sıra, günümüzün sosyal deneyimlerinin önemli bir parçası olan ziyaretçiler arasındaki bağlantıyı güçlendirmesi beklenmektedir. Kültürel miras bağlamında 3B baskı, artırılmış gerçeklik ve web tabanlı görselleştirme yoluyla değer yaratma fikrini niteliksel olarak keşfetmek için daha fazla araştırmaya ihtiyaç vardır. Bu nedenle, bu fikrin tüm potansiyelini keşfetmek için çok çeşitli paydaşlarla birlikte ortak çalışmaların yürütülmesi önerilmektedir.

\section{Kaynakça}

Aziz, K. A., \& Siang, T. G. (2014). Virtual Reality and Augmented Reality combination as a holistic application for heritage preservation in the Unesco World Heritage Site of Melaka. International Journal of Social Science and Humanity, 4(5), 333-338.

Bostanci, E., Kanwal, N., \& Clark, A. F. (2015). Augmented reality applications for cultural heritage using Kinect. Human-centric Computing and Information Sciences, 5(1), 118.

Bozzelli, G., Raia, A., Ricciardi, S., De Nino, M., Barile, N., Perrella, M., \& Palombini, A. (2019). An integrated VR/AR framework for user-centric interactive experience of cultural heritage: The ArkaeVision project. Digital Applications in Archaeology and Cultural Heritage, 15, e00124.

Fernández-Palacios, B. J., Morabito, D., \& Remondino, F. (2017). Access to complex reality-based 3D models using virtual reality solutions. Journal of cultural heritage, 23, 40-48.

Jung, T. H., \& tom Dieck, M. C. (2017). Augmented reality, virtual reality and $3 \mathrm{D}$ printing for the co-creation of value for the visitor experience at cultural heritage places. Journal of Place Management and Development, 10(2).

Nishanbaev, I. (2020). A web repository for geo-located 3D digital cultural heritage models. Digital Applications in Archaeology and Cultural Heritage, 16, e00139.

Putra, E. Y., Wahyudi, A. K., \& Dumingan, C. (2016, October). A proposed combination of photogrammetry, Augmented Reality and Virtual Reality Headset for heritage visualisation. In 2016 International Conference on Informatics and Computing (ICIC) (pp. 43-48). IEEE.

Uslu, A. ve Uysal, M. (2017). E-müze için kültürel mirasın 3 boyutlu modellenmesi ve gösterimi. Afyon Kocatepe
Üniversitesi Fen ve Mühendislik Bilimleri Dergisi. (17). 7985.

Uslu, A. ve Uysal, M. (2017). Arkeolojik Eserlerin Fotogrametri Yöntemi ile 3 Boyutlu Modellenmesi: Demeter Heykeli Örneği. Geomatik, 2(2), 60-65.

Uslu, A. ve Uysal, M. (2020). Kültürel Mirasın Etkileşimli Keşfi İçin Mobil Artırılmış Gerçeklik ve Web Tabanlı Görselleştirme Teknolojilerinin Kullanılması: Sfenks Heykeli Örneği. Afyon Kocatepe Üniversitesi Fen Ve Mühendislik Bilimleri Dergisi, 20 (6), 1024-1031.

Uslu, A. ve Uysal, M. (2021) Kitle Kaynaklı Fotoğraflar Kullanılarak Kültürel Mirasın Üç Boyutlu Modellenmesi ve Web Tabanlı Görselleştirilmesi: Afrodisias-Tetrapylon Örneği. Afyon Kocatepe Üniversitesi Fen Ve Mühendislik Bilimleri Dergisi, 21(3), 632-639.

Verim, Y. (2019). Kütahya'da II. Abdülhamid Han Dönemi'nde Inşa Edilmiş Iki Çeşme: Çinili Çeşme ve Hürriyet Çeşmesi. Safran Kültür ve Turizm Araştırmaları Dergisi, 2(1), 75-92.

URL-1: https://www.augment.com/ (Erişim Tarihi: 02.10.2021).

URL-2: https://cesium.com/platform/cesiumjs/ (Erişim Tarihi: 05.10.2021). 